\title{
Response to Comment on: Sector retinitis pigmentosa caused by mutations of the RHO gene
}

\author{
Ting Xiao' ${ }^{1}$ Yang $\mathrm{Li}^{1}$
}

Received: 24 September 2019 / Accepted: 24 September 2019 / Published online: 28 October 2019

(C) The Royal College of Ophthalmologists 2019

\section{To the Editor:}

We would like to thank Orlans and MacLaren for their attention to our recent paper "sector retinitis pigmentosa caused by mutations of the RHO gene" [1] and their suggestions on counseling patients who have sector retinitis pigmentosa (sector RP) due to the RHO mutation.

In their recent study, Orlans et al. found that retinal degeneration in RhoP23H/+ mice was significantly slower when the mice were housed in red-tinted cages than in untinted cages [2]. Some studies have also shown that retinal damage in rhodopsin-related RP animal models was more sensitive to light and that the progression of retinal degeneration was slower in dark environments $[3,4]$.

At present, no clinical trial data are available regarding the use of light filters as a treatment for patients with sector RP whose disease is caused by RHO mutations. A red filter could indeed be a potential cost-saving and noninvasive strategy that might delay or even prevent the progression of retinal degeneration in patients with sector RP due to RHO mutations. In clinical consultations with these patients with RHO mutations, doctors might consider advising the patients to start using physical protection as early as possible by wearing attenuated sunglasses or red-tinted eyeglasses when exposed to high outdoor light intensities. The doctors should also remind their patients about the possibility of experiencing temporary color vision changes and increased visual acuity defects in scotopic environments when wearing these types of glasses. Similarly, doctors should remain aware that they should avoid taking serial blue autofluorescence images in these patients to reduce the possibility of retinal damage caused by short-wavelength light. We also hope that relevant clinical trials will be developed and performed to explore optimal lens parameters, treatment time windows, treatment effects, possible side effects, and patient feedback.

\section{Compliance with ethical standards}

Conflict of interest The authors declare that they have no conflict of interest.

Publisher's note Springer Nature remains neutral with regard to jurisdictional claims in published maps and institutional affiliations.

\section{References}

1. Xiao T, Xu K, Zhang X, Xie Y, Li Y. Sector retinitis pigmentosa caused by mutations of the RHO gene. Eye. 2019;33:592-9.

2. Orlans HO, Merrill J, Barnard AR, Issa PC, Peirson SN, Maclaren RE. Filtration of short-wavelength light provides therapeutic benefit in retinitis pigmentosa caused by a common rhodopsin mutation. Investig Ophthalmol Vis Sci. 2019;60:2733-42.

3. Tam BM, Moritz OL. Dark rearing rescues $\mathrm{P} 23 \mathrm{H}$ rhodopsininduced retinal degeneration in a transgenic Xenopus laevis model of retinitis pigmentosa: a chromophore-dependent mechanism characterized by production of $\mathrm{N}$-terminally truncated mutant rhodopsin. J Neurosci. 2007;27:9043-53.

4. Iwabe S, Ying GS, Aguirre GD, Beltran WA. Assessment of visual function and retinal structure following acute light exposure in the light sensitive T4R rhodopsin mutant dog. Exp Eye Res. 2016;146:341-53.
Yang Li

yanglibio@aliyun.com

1 Beijing Institute of Ophthalmology, Beijing Tongren Eye Center, Beijing Tongren Hospital, Capital Medical University, Beijing Ophthalmology \& Visual Sciences Key Lab, Beijing, China 